Objective Paediatric Assessment Illness Resuscitation and Stabilisation Course (PAIRS) is an accredited one day course developed in Dublin for resource limited settings. The aim of this project was to deliver this course to front line paediatric staff in a south eastern Nigerian hospital.

Methods The course was delivered over 8 hours daily for 3 days with breaks in-between. On the 4th day, a number of delegates including doctors and senior nurses who had attended one of the 3 days were invited to participate in a more intensive course to develop more advanced skills in paediatric resuscitation and management of sick children. Delegates' knowledge was tested and they received certificates of completion at the end of the course.

Results Over the 4 days, 214 paediatric healthcare workers (136 doctors, 77 nurses, 1 'other') representing 21 hospitals were trained on a structured approach in the assessment and resuscitation of serious illness in children, team work and communication, paediatric early warning system and basic life support. Delegates reported increased knowledge and competency in the management of paediatric emergencies.

Conclusion The World Health Organisation (WHO) estimates that about five children under 5 years of age die in Africa every minute. More than half of these child deaths are due to conditions that are preventable or treatable with simple interventions. Teaching paediatric resuscitation enhances the skills of paediatric responders and has been shown to directly reduce morbidity and improve patient survival. This course enhanced their knowledge and helped the participants formulate tools required for early recognition and monitoring of illness progression in paediatric patients. On completion of the course, a local faculty was also instated to commence simulation training in the local hospital with support from the Ireland faculty. The training was well received and had a lot of positive feedback.

\section{G461(P) IF THE KAP FITS - KNOWLEDGE, ATTITUDE AND PRACTICE TOWARDS NEONATAL HEALTH IN RURAL CAMBODIA}

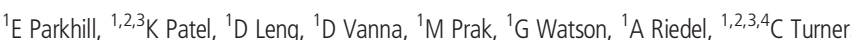
${ }^{1}$ Global Child Health Department, Angkor Hospital for Children, Siem Reap, Cambodia; ${ }^{2}$ Mahidol Oxford Tropical Medicine Research Unit, Mahidol University, Bangkok, Thailand; ${ }^{3}$ Cambodia Oxford Medical Research Unit, Angkor Hospital for Children, Siem Reap, Cambodia; ${ }^{4}$ Centre for Tropical Medicine and Global Health, University of Oxford, Oxford, UK

\subsection{6/archdischild-2020-rcpch.397}

Introduction Cambodia has one of the highest neonatal mortality rates (NMR) in Southeast Asia: 18 per 1,000 births. Most neonates die in rural areas, often in the community, and before reaching a health facility. Community Health Workers (CHWs) constitute a vital part of the national health system, providing health education and advice to their village. This reported study is a component of the five year 'Saving Babies' Lives' (SBL) programme, which aims to reduce NMR by implementing a comprehensive neonatal healthcare package in a rural Cambodian province. The programme intervention is participant action research to empower CHWs. The programme required a situation analysis of baseline CHW knowledge, attitude and practices, and to monitor temporal change; no SBL intervention had occurred during the timeframe examined.
Aim To examine the results and application of a neonatal Knowledge Attitudes and Practice (KAP) survey, for CHWs in a rural province in Cambodia, between 2018 and 2019.

Methods A bespoke neonatal CHW assessment tool, using a KAP survey method and based on national guidelines, was developed into a tablet-based survey using KoBoToolbox. The KAP survey comprised 47 grouped knowledge yes/no/ unsure questions, and seven attitude and six practice questions using a 5-point Likert scale. In August 2018 and July 2019 all CHWs attending existing monthly meetings were surveyed.

Results 420 CHWs were surveyed in 2018 and 375 in 2019. $87 \%$ of knowledge questions were correctly answered in 2018 , and $88 \%$ in 2019. Mean attitude scores were similar in 2018 and 2019 (3.95 and 3.87 respectively).Mean practice scores were also similar (2018: 4.46; 2019: 4.30).

Limitations in interpreting results include CHWs conferring during survey completion, and some confusingly worded questions.

Conclusion High baseline KAP scores possibly reflect the high volume of NGO-led CHW education in Cambodia. High scores mean change over time is unlikely to be captured, so an annual CHW KAP survey will be discontinued for the SBL programme. KAP surveys are a useful tool for situation analysis and to measure temporal changes. This study shows that the use of the KAP survey needs to be assessed and its use adapted according to findings.

\section{G462(P) ABSTRACT WITHDRAWN}

\section{G463(P) ABSTRACT WITHDRAWN}

\section{G464(P) BUILDING REFERRAL MECHANISMS FOR NEWBORN CARE IN HUMANITARIAN EMERGENCY SETTINGS: A SYSTEMATIC REVIEW}

${ }^{1} \mathrm{~S}$ Rutherford, ${ }^{2} \mathrm{~N}$ Zaka, ${ }^{3} \mathrm{~K}$ Leak, ${ }^{1} \mathrm{M}$ Cupp, ${ }^{1} \mathrm{H}$ Hafezi, ${ }^{4} \mathrm{E}$ Alexander, ${ }^{1} \mathrm{M}$ Lakhanpaul, ${ }^{5} \mathrm{~L}$ Manikam. ${ }^{1}$ Population, Policy and Practice, UCL, London, UK; ${ }^{2}$ Maternal and Newborn Health Team, UNICEF, New York, New York, US; ${ }^{3}$ Social Sciences, Social Statistics and Demography, University of Southampton, Southampton, UK; ${ }^{4}$ School of Medical Education, Kings College London, London, UK; ${ }^{5}$ Institute of Epidemiology and Health Care, UCL, London, UK

\subsection{6/archdischild-2020-rcpch.398}

Aim During humanitarian emergencies, women and children are particularly vulnerable to health complications, and neonatal mortality rates rise. Additionally, health cluster partners face challenges in coordinating referrals from communities and camps to health facilities, and between different levels of health facilities. The purpose of this review was to identify the primary referral needs among neonates during humanitarian emergencies, current gaps and barriers, and effective mechanisms for overcoming these barriers.

Methods A systematic review was performed using 4 electronic databases (CINAHL, EMBASE, Medline, and Scopus) and multiple grey literature sources between June and 
August 2019. Title, abstract, and full text screening were conducted following the Preferred Reporting Items for Systematic Reviews and Meta-Analyses. The target population was neonates born during humanitarian emergencies. Studies from high-income countries and prior to 1991 were excluded. The STROBE checklist was used to assess for risk of bias.

Results A total of 35 articles were included in the analysis; these were mainly qualitative, field-based studies. The primary needs identified were referrals from homes to health facilities before and during labour, and inter-facility referrals after labour to more specialised services. Some of the main barriers included a lack of roads and infrastructure for transport, a deficiency of specialised equipment for complications, staff shortages - especially among more specialised services, and a lack of knowledge among healthcare workers for identifying neonatal referral issues. Effective mechanisms for addressing these needs and gaps included establishing pre- or post-natal home visits by Community Health Workers or Traditional Birth Attendants; standardised, inter-facility referral checklists and procedures for healthcare staff; and staff training programmes for identification of neonatal complications. The severity and issues varied significantly between different countries and emergencies.

Conclusion This review benefited from a strong consensus among selected studies but was limited in the quality of data and types of data that were reported. Based on the above findings, the following recommendations were compiled:

- Recruit community healthcare workers to raise awareness and reduce pressure on health facilities.

- Establish contextualised programmes that address local needs and draw on local resources.

- Continue to train healthcare workers to provide timely, appropriate and quality care during humanitarian emergencies.

\section{G465(P) TACKLING LOCAL OBSTACLES TO EFFECTIVE KANGAROO MOTHER CARE IN A DISTRICT HOSPITAL IN RURAL RWANDA: AN OBSERVATIONAL STUDY}

${ }^{1} \mathrm{~T}$ Slater, ${ }^{2}$ E Smeaton-Russell, ${ }^{3} \mathrm{~T}$ Lissauer. ${ }^{1}$ Specialist Registrar, South Thames Deanery, London, UK; ${ }^{2}$ Paediatric Nurse, Great Ormond Street Hospital, London, UK; ${ }^{3}$ Hon. Consultant Neonatologist, Imperial College Healthcare Trust, London, UK

\subsection{6/archdischild-2020-rcpch.399}

Aims To identify obstacles to effective Kangaroo Mother Care (KMC) at a district hospital in rural Rwanda.

Background KMC can improve thermoregulation and weight gain, whilst reducing infections and ultimately mortality. It can provide a safe alternative to incubator care for stable babies. Whilst KMC is often an established facility in Rwanda hospitals, local barriers may exist to limit the desired practice and outcomes in infants.

Methods Over a 2-week period at the start of a 6-month placement, detailed observations of KMC practice 3 times a day were made.

Results were collated and discussed with medical and nursing staff working in the neonatal unit. A programme to overcome key, local barriers to effective KMC was then devised.
Results Key barriers and initiatives to overcome them were:

- Poor maternal nutrition - extreme local poverty and geographical isolation of the hospital on a mountain led to limited social support for mothers. Prolonged absence (and even absconding) of mothers was necessary to source food. Provision of a water filter and creation of a local egg cooperative helped to improve nutrition and minimise time needed away from their babies. With support for the cooperative from the adjacent church, free eggs are now provided daily to long-term mothers in the unit.

- Lack of space - a new, larger room for mothers undertaking KMC was sourced with our supervision, as part of a planned hospital re-organisation. This provided more space and independent washing facilities for mothers, creating a more pleasant environment.

- Lack of facilities -sourcing locally produced comfortable chairs and appropriate storage for belongings encouraged mothers to spend more effective time with their babies in KMC.

- Limited nursing staff - other members of the hospital staff, including social workers were mobilised to run education sessions on neonatal 'red flags' (i.e. fever, jaundice, lethargy, poor feeding). Basic educational materials and posters were provided to facilitate this.

Conclusion Detailed observation followed by staff engagement allowed many of the obstacles to effective KMC to be overcome with low cost interventions. Nevertheless, innovative and local initiatives are critical to achieving major improvements.

\section{G466(P) TRAINING ON THE RECOGNITION OF THE SICK NEONATE}

${ }^{1} \mathrm{E}$ MacRae, ${ }^{2} \mathrm{~T}$ Kyaw, ${ }^{3} \mathrm{SM}$ Thu, ${ }^{4} \mathrm{~L}$ Davidson. ${ }^{1}$ Paediatrics, RCPCH, London, UK; ${ }^{2}$ Paediatrics, Myanmar Paediatric Society, Hinthada, Myanmar; ${ }^{3}$ Paediatrics, Myanmar Paediatric Society, Mawgyun, Myanmar; ${ }^{4}$ Paediatric Nursing, Royal College of Nursing, London, UK

\subsection{6/archdischild-2020-rcpch.400}

Background As part of the partnership with Myanmar paediatric society and the Royal College of paediatrics, work has been undertaken to improve child and neonatal mortality. Our aim was to increase vital sign monitoring; the recognition of the sick or deteriorating neonate and timely escalation of care. To achieve this objective, a training programme was devised for nursing staff to increase awareness of neonatal danger signs and when to inform the doctors of the patient's condition.

Objective To determine if the training programme for nursing staff was effective in improving their awareness of neonatal danger signs and when to inform the doctors of the patient's condition. This was determined by comparing their mean test scores before and after the programme for any statistically significant improvement.

Method The training was carried out amongst 17 nurses (paediatric, neonatal and nursing students) in two hospitals in the Ayeyarwady region (Hospital A and Hospital B). A poster was created detailing how to monitor the neonates, basic emergency management of sick neonates for the nursing staff and when to escalate concerns to a paediatric doctor. 